\title{
Asymptotic Models for the Topological Sensitivity Versus the Topological Derivative
}

\author{
I.I. Argatov*
}

Research Institute of Mechanical Engineering Problems of the RAS, Russia

\begin{abstract}
An approach, based on the refined method of matched asymptotic expansions, is proposed for the construction of asymptotic models for the topological sensitivity of the energy functional with respect to the creation of a small hole in the geometrical domain. It is shown that the asymptotic model provides more information for calculations than the topological derivative.
\end{abstract}

\section{INTRODUCTION}

The shape optimization theory [1] provides well established techniques for the investigation of shape optimization problems when the topology class of the geometrical domain under consideration is supposed to be fixed. At the same time, the shape optimization methods cannot produce useful criteria whether a topological change (for instance, the creation of a hole in the interior of the geometrical domain) will lead to a decreasing value of the shape functional or not. One such criterion [2] is based on the notion of the topological derivative whose importance in the topology optimization is now widely recognized.

The present paper is devoted to analyzing the application of the topological derivative in shape and topology optimization problems which take into consideration the question of changing the topology class of the geometrical domain. More precisely, the so-called asymptotic model based on the refined asymptotic expansion is presented for the topological sensitivity of the Dirichlet integral in a special case of nucleation of a hole with the homogeneous Neumann boundary condition imposed on its boundary.

It is known that the aim of the topological sensitivity analysis (see, for example, [3,4]) is to obtain the so-called topological asymptotic expansion of a given shape functional $J(\Omega, v)$ with respect to the creation of a small opening $\omega_{\varepsilon}\left(x^{0}\right)$ of diameter $O(\varepsilon)$ with the center at a given point $x^{0}$ in the geometrical domain $\Omega(0<\varepsilon$ is a small parameter).

Let $T_{\omega}^{\varepsilon}\left(x^{0}\right)$ be the ratio between the difference $J\left(\Omega_{\varepsilon}, u^{\varepsilon}\right)-J\left(\Omega, v^{0}\right)$, where $u^{\varepsilon}$ is the solution of the boundary value problem defined on the singularly perturbed domain $\Omega_{\varepsilon}=\Omega \backslash \overline{\omega_{\varepsilon}\left(x^{0}\right)}$, and the area $\left|\omega_{\varepsilon}\left(x^{0}\right)\right|$ of the small opening $\omega_{\varepsilon}\left(x^{0}\right)$.

*Address correspondence to this author at the Laboratory of Friction and Wear, Research Institute of Mechanical Engineering Problems, V.O., Bolshoy pr., 61, 199178 St. Petersburg, Russia; E-mail: argatov@home.ru
We shall say that $T_{\omega}^{\varepsilon}\left(x^{0}\right)$ is the topological sensitivity of the shape functional $J(\Omega, v)$ with respect to the internal topological variation (i.e., creation of the small hole $\omega_{\varepsilon}\left(x^{0}\right)$ in the domain $\Omega$ ). The idea of topological sensitivity (the term has been used in a number of publications [3], etc.) was introduced in [2] (the so-called characteristic function) in the framework of the bubble method for topology and shape optimization in two-dimensional elastostatic problems.

In the case of the homogeneous Neumann boundary condition imposed on the boundary $\partial \omega_{\varepsilon}\left(x^{0}\right)$ of the hole $\omega_{\varepsilon}\left(x^{0}\right)$, the topological asymptotics for the energy functional can be obtained in the form $[5,3]$

$J\left(\Omega_{\varepsilon}, u^{\varepsilon}\right)=J\left(\Omega, v^{0}\right)+T_{\omega}^{0}\left(x^{0}\right)\left|\omega_{\varepsilon}\left(x^{0}\right)\right|+o\left(\left|\omega_{\varepsilon}\left(x^{0}\right)\right|\right)$.

Here, $T_{\omega}^{0}\left(x^{0}\right)$ is the topological derivative which determines whether a change of topology by nucleation of a small hole $\omega_{\varepsilon}\left(x^{0}\right)$ at the point $x^{0}$ in the interior of the domain $\Omega$ would result in improving the value of the shape functional $J(\Omega, v)$ or not.

The aim of the asymptotic modelling is to obtain a refined asymptotic representation for the topological sensitivity, i.e., for the increment of a given shape functional resulting from the emerging of a small opening in the interior of the domain. As a result of application of the refined method of matched asymptotic expansions [6,7], we obtain the following asymptotic formula:

$T_{\omega}^{\varepsilon}\left(x^{0}\right)=S_{\omega}^{\varepsilon}\left(x^{0}\right)+o\left(\left|\omega_{\varepsilon}\left(x^{0}\right)\right|\right), \quad \varepsilon \rightarrow 0$,

where $S_{\omega}^{\varepsilon}\left(x^{0}\right)$ is an asymptotic model for the topological sensitivity.

We stress that the so-called asymptotic model for the topological sensitivity $S_{\omega}^{\varepsilon}\left(x^{0}\right)$, which may be regarded as a Padé approximant [8], provides more information for calculations and they have more accuracy (see, e.g., $[9,7,10]$ ). 


\section{TOPOLOGICAL DERIVATIVE}

Let $\Omega$ and $\omega$ be two domains on $R^{2}$ with compact closures $\bar{\Omega}$ and $\bar{\omega}$ and smooth boundaries $\partial \Omega$ and $\partial \omega$. We assume that $\omega$ contains the origin $O$. By $\varepsilon$ we denote a small positive parameter. For small $\varepsilon$ it is possible for any fixed point $x^{0} \in \Omega$ to remove the set $\omega_{\varepsilon}\left(x^{0}\right)=\left\{x=\left(x_{1}, x_{2}\right) \mid \varepsilon^{-1}\left(x-x^{0}\right) \in \omega\right\}$ from $\Omega$, obtaining the singularly perturbed domain $\Omega_{\varepsilon}$ with the boundary $\partial \Omega_{\varepsilon}=\partial \Omega \cup \partial \omega_{\varepsilon}\left(x^{0}\right)$. In such a domain we consider the following mixed boundary value problem for the Poisson equation:

$-\Delta u^{\varepsilon}(x)=f(x), x \in \Omega_{\varepsilon} ; \quad u^{\varepsilon}(x)=0, x \in \partial \Omega ;$

$\partial_{n} u^{\varepsilon}(x)=0, x \in \partial \omega_{\varepsilon}\left(x^{0}\right)$.

Here, $\partial_{n}$ stands for the derivative in the direction of the outward (with respect to $\Omega_{\varepsilon}$ ) normal vector $n$.

In this paper the asymptotic behavior of the solution $u^{\varepsilon}(x)$ is considered and the leading terms of asymptotic expansions are constructed. The inclusion $f \in C^{1}(\bar{\Omega})$ is sufficient for our purposes.

As $\varepsilon \rightarrow 0$, the hole $\omega_{\varepsilon}\left(x^{0}\right)$ is collapsed to the point $x^{0}$, the boundary condition (2) disappears, and relations (1) form the limit problem

$-\Delta v^{0}(x)=f(x), x \in \Omega ; \quad v^{0}(x)=0, x \in \partial \Omega ;$

We consider the case of the energy functional

$J\left(\Omega, v^{0}\right)=\frac{1}{2} \int \nabla_{\Omega}\left|\nabla_{x} \nu^{0}(x)\right|^{2} d x$.

The topological derivative $T_{\omega}^{0}\left(x^{0}\right)$ of the functional $J\left(\Omega, v^{0}\right)$ at the point $x^{0} \in \Omega$ is defined by $[5,4]$

$T_{\omega}^{0}\left(x^{0}\right)=\lim _{\varepsilon \rightarrow 0+} \frac{J\left(\Omega_{\varepsilon}, u^{\varepsilon}\right)-J\left(\Omega, v^{0}\right)}{\left|\omega_{\varepsilon}\left(x^{0}\right)\right|}$.

By definition, we put

$T_{\omega}^{\varepsilon}\left(x^{0}\right)=\frac{J\left(\Omega_{\varepsilon}, u^{\varepsilon}\right)-J\left(\Omega, v^{0}\right)}{\left|\omega_{\varepsilon}\left(x^{0}\right)\right|}$,

where $T_{\omega}^{\varepsilon}\left(x^{0}\right)$ is the topological sensitivity.

The topological derivative (5) in the case (2) under the condition of a hole $\omega_{\varepsilon}\left(x^{0}\right)$ being a ball in $R^{2}$ was introduced in [5] with a reference to the original idea by Schumacher (1995), suggested for the special case of the energy functional in linear elasticity in the framework of the bubble method [2]. The topological derivative concept was latter generalized for nucleation of cavities of arbitrary shape
$[3,11]$ as well, as for the cases of different types of boundary conditions imposed on $\partial \omega_{\varepsilon}\left(x^{0}\right)$ and different state differential equations defined on $\Omega_{\varepsilon} \in R^{n} \quad(n=1,2)[4,12]$. Also, different approaches were suggested for calculation of the topological derivative $[3,13,4]$. Let us emphasize [14] that the topological derivatives can be obtained in a different way, but the forms of such derivatives are equivalent.

The methods of topology optimization based on the bubble method are used for the topology optimization in structural mechanics $[2,15]$. Numerical results obtained by help of the topological derivative can be found in $[3,12]$. We refer to $[16,17]$ for applications in inverse problems. The topological derivative was incorporated [18] into the level set method [19]. Note that asymptotic models can be connected, in particular, with the primal-dual active set method for crack problems with non-penetration [20] as a criteria for the kinking of a crack. Note also that asymptotic models for dilute and densely packed composites [21] and asymptotic solutions for periodic problems obtained in [22] could be used in the homogenization method for shape and topology optimization [23].

In [14] the case of a finite number of circular holes was treated by means of the so-called topological gradient which contains the topological derivatives evaluated at the centers of holes. In [24] two new approaches were proposed for the modelling of so-called internal multiple topological variations. The first approach is developed in the framework of the self adjoint extensions of differential operators, the second is based on the variational formulation with point asymptotic conditions in a functional space with separated asymptotics. In the present paper the third approach is proposed for the asymptotic modeling of the internal topological variations. This approach is based on the refined method of matched asymptotic expansions [6,7]. Namely, the refined method of matched expansions in the form [7] is applied to construction of asymptotic models for the topological sensitivity of the energy functional.

To this end, Sections 1 and 2 briefly recall the notion of topological derivative, evaluation of the topological derivative by help of the method of matched asymptotic expansions and its refined modification [7]. The performed asymptotic analysis is formal. Estimates for the proposed approximations in weighted Hölder spaces [4] and weighted Sobolev spaces [25] were derived in the context of shape optimization.

The asymptotics of the solution to the singularly perturbed problem (1), (2) is known (see, [26]). We use the method of matched asymptotic expansions [27,28]. This method implies the following structure for the inner asymptotic expansion:

$u^{\varepsilon}(x)=w^{0}(\xi)+\varepsilon w^{1}(\xi)+\ldots$,

which is valid in a small neighborhood of $\omega_{\varepsilon}\left(x^{0}\right)$. In (7), we introduced the so-called stretched variables

$\xi=\left(\xi_{1}, \xi_{2}\right), \quad \xi_{i}=\varepsilon^{-1}\left(x_{i}-x_{i}^{0}\right)$. 
Changing to the stretched coordinates (8) in (1) and (2), after passage to the limit $\varepsilon=0$, we conclude that $w^{q}(\xi)$ ( $q=0,1$ ) must satisfy the following relations:

$\Delta_{\xi} w^{q}(\xi)=0, \xi \in R^{2} \backslash \bar{\omega} ; \quad \partial_{v} w^{q}(\xi)=0, \xi \in \partial \omega$.

The Dirichlet boundary condition (1) is replaced with an asymptotic condition restricting the behavior of $w^{q}(\xi)$ as $\xi \rightarrow \infty$. That asymptotic condition results from matching with the solution $v^{0}(x)$ of the limit problem (3). The matching procedure $[27,28]$ implies that the leading terms in the asymptotic expansions for $v^{0}(x)$ and $w^{0}(\xi)+\varepsilon w^{1}(\xi)$ formally coincide as $x \rightarrow 0$ and $\xi \rightarrow \infty$, respectively.

Consequently, in view of the Taylor formula

$v^{0}(x)=v^{0}\left(x^{0}\right)+\left(x-x^{0}\right) \nabla v^{0}\left(x^{0}\right)+O\left(\left|x-x^{0}\right|^{2}\right)$,

we add to the second limit problem (9), $q=1$, the asymptotic condition $w^{1}(\xi)=\xi \nabla v^{0}\left(x^{0}\right)+O\left(|\xi|^{-1}\right)$ as $\xi \rightarrow \infty$. Hence, $w^{1}(\xi)$ can be represented as

$w^{1}(\xi)=\sum_{l=1,2} \frac{\partial v^{0}}{\partial x_{l}}\left(x^{0}\right) Y^{l}(\xi)$

Here, $Y^{L}(\xi)=\xi_{L}+Y_{0}^{L}(\xi)(l=1,2)$ are the special solutions to the exterior Neumann problem (9) that admit the following asymptotic expansions:

$Y_{0}^{l}(\xi)=\frac{1}{2 \pi|\xi|^{2}} \sum_{k=1,2} m_{k l}(\omega) \xi_{k}+O\left(|\xi|^{-2}\right)$.

The outer asymptotic expansion for the solution $u^{\varepsilon}(x)$ to our problem (1), (2) has the form

$u^{\varepsilon}(x)=v^{0}(x)+\varepsilon^{2} v^{1}(x)+\ldots$.

In view of (11) and (12), the function $v^{1}(x)$ must satisfy the following asymptotic condition $\left(x \rightarrow x^{0}\right)$ :

$v^{1}(x)=\sum_{l, k=1}^{2} m_{k l}(\omega) \frac{\partial v^{0}}{\partial x_{l}}\left(x^{0}\right) \frac{x_{k}-x_{k}^{0}}{2 \pi\left|x-x^{0}\right|^{2}}+O(1)$.

We denote by $G^{(k)}\left(x^{0}, x\right) \quad(k=1,2)$ singular solutions to the homogeneous problem (1) that admit the following representation:

$G^{(k)}\left(x^{0}, x\right)=\frac{x_{k}-x_{k}^{0}}{2 \pi\left|x-x^{0}\right|^{2}}+g^{(k)}\left(x^{0}, x\right)$.

In accordance with (14), we obtain

$v^{1}(x)=\sum_{l, k=1}^{2} m_{k l}(\omega) \frac{\partial v^{0}}{\partial x_{l}}\left(x^{0}\right) G^{(k)}\left(x^{0}, x\right)$, where $m(\omega)$ is the matrix consisting of the coefficients from the asymptotic formula (12).

Note that the obtained asimptotics (13), (16) can be derived by the method of compound asymptotic expansions [26] or the asymptotic method [29] based on layer potential techniques (see, formula (4.16)).

An asymptotic approximation of the functional $J\left(\Omega_{\varepsilon}, u^{\varepsilon}\right)$ can be derived from formulas (4) and (11), (16). However, using Green's formula, we obtain

$J\left(\Omega_{\varepsilon}, u^{\varepsilon}\right)=\frac{1}{2} \int_{\Omega_{\varepsilon}} f(x) u^{\varepsilon}(x) d x$.

Here, the homogeneous Dirichlet boundary condition (1) on $\partial \Omega$ and the Neumann boundary condition (2) on $\partial \omega_{\varepsilon}\left(x^{0}\right)$ were taken into account.

The following two-term asymptotic expansion of the energy functional is valid (see, e.g., [26,31,32]):

$$
\begin{aligned}
J\left(\Omega_{\varepsilon}, u^{\varepsilon}\right) & =J\left(\Omega, v^{0}\right)-\frac{\varepsilon^{2}}{2} v^{0}\left(x^{0}\right) f^{0}\left(x^{0}\right)|\omega| \\
& +\frac{\varepsilon^{2}}{2} \nabla v^{0}\left(x^{0}\right)^{\mathrm{T}} m(\omega) \nabla v^{0}\left(x^{0}\right)+o\left(\varepsilon^{2+\sigma}\right),
\end{aligned}
$$

where $\sigma>0$ is small.

Hence, since $\left|\omega_{\varepsilon}\left(x^{0}\right)\right|=\varepsilon^{2}|\omega|$, the following value of the topological derivative is obtained:

$$
\begin{aligned}
T_{\omega}\left(x^{0}\right) & =-\frac{1}{2} v^{0}\left(x^{0}\right) f^{0}\left(x^{0}\right) \\
& +\frac{1}{2|\omega|} \nabla v^{0}\left(x^{0}\right)^{\mathrm{T}} m(\omega) \nabla v^{0}\left(x^{0}\right) .
\end{aligned}
$$

Here, $m(\omega)$ is a symmetric positive definite $2 \times 2$ matrix associated with the virtual mass tensor [30]. Following $[4,22]$, we call $m(\omega)$ the polarization matrix.

\section{ASYMPTOTIC MODEL}

We use the refined method of matched asymptotic expansions in the form [7]. In view of (13) and (16) we take the sum

$$
V^{\varepsilon}(x)=v^{0}(x)+\varepsilon^{2} \sum_{k=1}^{2} C_{k} G^{(k)}\left(x^{0}, x\right)
$$

as the outer asymptotic representation of the solution $u^{\varepsilon}(x)$ to the singularly perturbed problem (1), (2).

By formulas (10) and (15) we have

$$
\begin{aligned}
V^{\varepsilon}(x) & =v^{0}\left(x^{0}\right)+\varepsilon^{2} \sum_{k=1,2} C_{k} g_{0}^{(k)}+\Delta x \nabla v_{0}^{0} \\
& +\varepsilon^{2} \sum_{k=1}^{2} C_{k}\left\{\frac{\Delta x_{k}}{2 \pi|\Delta x|^{2}}+\Delta x \nabla g_{0}^{(k)}\right\}+O\left(|\Delta x|^{2}\right) ; \\
\nabla g_{0}^{(k)} & =\nabla g^{(k)}\left(x^{0}, x^{0}\right), \nabla v_{0}^{0}=\nabla v^{0}\left(x^{0}\right), \Delta x=x-x^{0} .
\end{aligned}
$$



tain

Substituting the stretched coordinates (8) in (21), we ob-

$$
\begin{aligned}
V^{\varepsilon}(x) & =v^{0}\left(x^{0}\right)+\varepsilon^{2} \sum_{k=1,2} C_{k} g_{0}^{(k)}+\varepsilon \xi \nabla v_{0}^{0} \\
& +\varepsilon^{2} \sum_{k=1}^{2} C_{k}\left\{\frac{\xi_{k}}{\varepsilon 2 \pi|\xi|^{2}}+\varepsilon \xi \nabla g_{0}^{(k)}\right\}+O\left(\varepsilon^{2}|\xi|^{2}\right) .
\end{aligned}
$$

Following [7], we derive from (22) the refined matching asymptotic condition

$$
\begin{aligned}
W^{\varepsilon}(\xi) & =v^{0}\left(x^{0}\right)+\varepsilon^{2} \sum_{k=1,2} C_{k} g_{0}^{(k)} \\
& +\varepsilon \xi\left(\nabla v_{0}^{0}+\varepsilon^{2} \sum_{k=1}^{2} C_{k} \nabla g_{0}^{(k)}\right)+O\left(|\xi|^{-1}\right) .
\end{aligned}
$$

Hence, the inner asymptotic representation, which satisfies the second limit problem (9), (23), has the form $W^{\varepsilon}(\xi)=w^{0}(\xi)+\varepsilon w^{1}(\xi)$, where

$w^{1}(\xi)=\sum_{l=1}^{2}\left(\frac{\partial v^{0}}{\partial x_{l}}\left(x^{0}\right)+\varepsilon^{2} \sum_{k=1}^{2} C_{k} \frac{\partial g^{(k)}}{\partial x_{l}}\left(x^{0}\right)\right) Y^{l}(\xi)$.

Introducing the column of solutions $Y=\left(Y^{1}, Y^{2}\right)^{\mathrm{T}}$, where $\mathrm{T}$ denotes the transpose, and the symmetric $2 \times 2$ matrix $\nabla g_{0}^{(\bullet)}$ with the elements $\partial_{x_{l}} g^{(k)}\left(x^{0}, x^{0}\right) \quad(k, l=1,2)$, we rewrite (24) in the form

$w^{1}(\xi)=\left(\nabla v_{0}^{0}+\varepsilon^{2} \nabla g_{0}^{(\bullet)} C\right)^{\mathrm{T}} Y(\xi)$,

where $C=\left(C_{1}, C_{2}\right)^{\mathrm{T}}$.

Making use of the relations (12) we obtain

$w^{1}(\xi) \cong\left(\xi+\frac{1}{2 \pi|\xi|^{2}} \xi m(\omega)\right)\left(\nabla v_{0}^{0}+\varepsilon^{2} \nabla g_{0}^{(\bullet)} C\right)$,

On the other hand, from (22) we derive

$V^{\varepsilon}(x)-w^{0} \cong \varepsilon \xi\left(\nabla v_{0}^{0}+\varepsilon^{2} \nabla g_{0}^{(\bullet)} C\right)+\frac{\varepsilon}{2 \pi|\xi|^{2}} \xi C$.

Comparing (26) and (27), we obtain the equation

$C=m(\omega)\left(\nabla v_{0}^{0}+\varepsilon^{2} \nabla g_{0}^{(\bullet)} C\right)$.

From (28) we readily find

$C=\left(I-\varepsilon^{2} m(\omega) \nabla g_{0}^{(\bullet)}\right)^{-1} m(\omega) \nabla v_{0}^{0}$,

where $I=\operatorname{diag}\{1,1\}$.

Note that, first introduced in [6], the specific matrix notation used in [4] for asymptotic analysis of various shape functionals also provides asymptotic representations essentially similar to (25) and (20), (29) as it has been comprehensively shown in [33]. The refined method of matched asymptotic expansions [6,7] was applied for construction of asymptotic models in contact mechanics [33,34], in the theory of cracks [35], and in the theory of acoustic diffraction [36].
In order to evaluate an asymptotic approximation of the energy functional (4), we construct the uniformly suitable asymptotic representation

$$
\begin{aligned}
U^{\varepsilon}(x) & =v^{0}(x)+\varepsilon^{2} \sum_{k=1,2} C_{k} G^{(k)}\left(x^{0}, x\right) \\
& +\varepsilon \sum_{l=1,2}\left\{\frac{\partial v^{0}}{\partial x_{l}}\left(x^{0}\right)+\varepsilon^{2} \sum_{k=1}^{2} C_{k} \frac{\partial g^{(k)}}{\partial x_{l}}\left(x^{0}\right)\right\} \times \\
& \times\left(Y_{0}^{l}\left(\frac{x-x^{0}}{\varepsilon}\right)-\varepsilon \sum_{k=1,2} m_{k l}(\omega) \frac{x_{l}-x_{l}^{0}}{2 \pi\left|x-x^{0}\right|^{2}}\right)
\end{aligned}
$$

where the coefficients $C_{k}$ are defined by (29).

Thus, replacing the function $u^{\varepsilon}(x)$ with its asymptotics (30) in the integral (17), we obtain

$$
J\left(\Omega_{\varepsilon}, u^{\varepsilon}\right)=J\left(\Omega, v^{0}\right)+S_{\omega}^{\varepsilon}\left(x^{0}\right)\left|\omega_{\varepsilon}\left(x^{0}\right)\right|+o\left(\varepsilon^{2+\sigma}\right),
$$

where $\sigma>0$ is small.

Taking into account (29) and (31), we find

$$
\begin{aligned}
S_{\omega}^{\varepsilon}\left(x^{0}\right) & =-\frac{1}{2} v^{0}\left(x^{0}\right) f\left(x^{0}\right) \\
& +\frac{1}{2|\omega|}\left(\nabla v_{0}^{0}\right)^{\mathrm{T}}\left(I-\varepsilon^{2} m(\omega) \nabla g_{0}^{(\bullet)}\right)^{-1} m(\omega) \nabla v_{0}^{0} .
\end{aligned}
$$

Note that, since $\left(I-\varepsilon^{2} m(\omega) \nabla g_{0}^{(\bullet)}\right)^{-1}=I+O\left(\varepsilon^{2}\right)$, the asymptotic expansion (18) follows from (31). The proof uses the same tools as for the case (18). Note also that $S_{\omega}^{\varepsilon}\left(x^{0}\right)=T_{\omega}^{0}\left(x^{0}\right)+O\left(\varepsilon^{2}\right)$ as $\varepsilon \rightarrow 0$.

We emphasize that the formulas (18) and (31) are known to possess the same asymptotic accuracy, since both inner asymptotic representations (7) and (25) do not eliminate the discrepancy in the boundary condition (2) on $\partial \omega_{\varepsilon}\left(x^{0}\right)$ left by the term $O\left(\left|x-x^{0}\right|^{2}\right)$ from the expansion (10). However, the refined asymptotic representation (31) gives in fact better results because its construction is obtained by summation of asymptotic terms corresponding to the term $O\left(\left|x-x^{0}\right|\right)$.

The following asymptotic formula for the energy increment due to appearing of the cavity $\omega_{\varepsilon}\left(x^{0}\right)$ in the domain $\Omega$ is obtained (see, formula (31)):

$\frac{J\left(\Omega_{\varepsilon}, u^{\varepsilon}\right)-J\left(\Omega, v^{0}\right)}{\left|\omega_{\varepsilon}\left(x^{0}\right)\right|} \cong S_{\omega}^{\varepsilon}\left(x^{0}\right), \quad \varepsilon \rightarrow 0$.

Thus, we have $T_{\omega}^{\varepsilon}\left(x^{0}\right) \cong S_{\omega}^{\varepsilon}\left(x^{0}\right)$ as $\varepsilon \rightarrow 0$ (see, (6)), where $T_{\omega}^{\varepsilon}\left(x^{0}\right)$ is the topological sensitivity and $S_{\omega}^{\varepsilon}\left(x^{0}\right)$ is its asymptotic model of the first order.

\section{CONCLUSIONS}

Let us point out that the difference between the asymptotic model (32) for the topological sensitivity $T_{\omega}^{\varepsilon}\left(x^{0}\right)$ and 
the topological derivative $T_{\omega}^{0}\left(x^{0}\right)$ is substantial. First, the asymptotic model (33), (32) provides more information, since it takes into account the influence of a hole $\omega_{\varepsilon}\left(x^{0}\right)$ on the solution $u^{\varepsilon}(x)$ of the perturbed problem (1), (2), whereas the topological derivative (5), (19) depends only on the solution $v^{0}(x)$ of the non-perturbed problem (3). This additional information is contained in the matrix $\nabla g_{0}^{(\bullet)}$ whose components are defined by the geometry of the domain $\Omega$ and depend on the location of the point $x^{0}$. In other words, unlike the topological derivative, the asymptotic model for the topological sensitivity $S_{\omega}^{\varepsilon}\left(x^{0}\right)$ provides essential information of the response caused by the creation of a cavity $\omega_{\varepsilon}\left(x^{0}\right)$ in the geometrical domain $\Omega$.

Second, the asymptotic model (32) for the topological sensitivity $S_{\omega}^{\varepsilon}\left(x^{0}\right)$ defined by (33) may be regarded as a Padé approximant (see, e.g., [8]). A relation between the Padé approximation and the refined method of matched asymptotic expansions was established in [7]. Namely, this relation explains a surprising increasing of accuracy of asymptotic representations such as (32) for $\varepsilon \in\left(0, \varepsilon_{0}\right)[7,10]$.

Third, the asymptotic model based topological sensitivity is more sensitive tool in obtaining the optimal topology in the problem under consideration. In fact, using the asymptotic model (33), (32), it is possible to consider differences between points with the same value of the topological derivative. On the other hand, the topological derivative is a less sensitive tool because its value is obtained evaluating the limit $T_{\omega}^{0}\left(x^{0}\right)=\lim _{\varepsilon \rightarrow 0+} S_{\omega}^{\varepsilon}\left(x^{0}\right)$. We stress that the topological derivative gives a possibility for insertion of an infinitesimally small hole, whereas the bubble method $[2,15]$ requires insertion of a hole of small but finite size.

Fourth, one of the main advantages of the asymptotic model for the topological sensitivity is that it provides a more accurate tool for identification of cracks and cavities in the inverse problems (see, e.g., $[16,17]$ ). At the same time, the asymptotic model based topological sensitivity $S_{\omega}^{\varepsilon}\left(x^{0}\right)$ defined by (32) is computed using only information of the non-damaged domain $\Omega$.

The main results of the paper were reported for the first time in November 2004 on the research seminar at the Institute of Mathematics of the University of Graz (Austria). The author is grateful to Professor K. Kunisch and Dr. V.A. Kovtunenko for valuable discussions.

\section{REFERENCES}

[1] Sokolowski J, Zolesio JP. Introduction to shape optimization: shape sensitity analysis. Springer Series in Computational Mathematics, Springer, Berlin 1992; Vol. 10.

[2] Eschenauer HA, Kobelev VV, Schumacher A. Bubble method for topology and shape optimization of structures. Struct Optimiz 1994; 8: 42-51.

[3] Garreau S, Guillaume P, Masmoudi M. The topological asymptotic for PDE systems: The elasticity case. SIAM J Control Optim 2001; 39: $1756-78$.
[4] Nazarov SA, Sokolowski J. Asymptotic analysis of shape functionals. J Math Pures Appl 2003; 82: 125-96.

[5] Sokolowski J, Zochowski A. On the topological derivative in shape optimization. SIAM J Control Optim 1999; 37: 1251-72.

[6] Nazarov SA. Asymptotic conditions at points, selfadjoint extensions of operators and the method of matched asymptotic expansions. Trans Amer Math Soc Ser 2 1999; 193: 77-126.

[7] Argatov II. Refinement of the asymptotic solution obtained by the method of matched expansions in contact problem of elasticity theory. Comput Math Math Phys 2000; 40: 594-603.

[8] Baker GA, Graves-Morris P. Padé Approximants, Cambridge Univ Press, Cambridge 1996.

[9] Van Dyke MD. Analysis and improvement of perturbation series. Quart J Mech Appl Math 1974; 27: 423-50.

[10] Andrianov IV, Awrejcewicz J. Asymptotic approaches in mechanics: New parameters and procedures. Appl Mech Rev 2003; 56: 87110 .

[11] Lewinski T, Sokolowski J. Energy change due to appearing of cavities in elastic solids. Intern J Solids Struct 2003; 40: 17651803.

[12] Guillaume $\mathrm{Ph}$, Sid Idris K. The topological asymptotic expansion for the Dirichlet problem. SIAM J Control Optim 2002; 41: 104272.

[13] Novotny AA, Feijóo RA, Taroco E, Padra C. Topological sensitivity analysis. Comput Methods Appl Mech Eng 2003; 192: 803-29.

[14] Sokolowski J, Żochowski A. Optimality conditions for simultaneous topology and shape optimization. SIAM J Control Optim 2003; 42: 1198-221.

[15] Tai K, Fenner RT. Optimum shape and topology design using the boundary element method. Intern J Solids Struct 1999; 36: 202140 .

[16] Sokolowski J, Żochowski A. Topological derivatives for elliptic problems. Inverse Problems 1999; 15: 123-34.

[17] Gallego R, Rus G. Identification of cracks and cavities using the topological sensitivity boundary integral equation. Comput Mech 2004; 33: 154-63.

[18] Burger M, Hackl B, Ring W. Incorporating topological derivatives into level set methods. J Comput Phys 2004; 194: 344-62.

[19] Osher S, Fedkiw RP. Level set methods: an overview and some recent results. J Comput Phys 2001; 169: 463-502.

[20] Hintermüller M, Kovtunenko VA, Kunisch K. The primal-dual active set method for a crack problem with non-penetration. IMA J Appl Math 2004; 69: 1-26.

[21] Movchan AB, Movchan NV, Poulton CG. Asymptotic models of fields in dilute and densely packed composites. Imperial College Press, Liverpool 2002.

[22] Zhikov VV, Kozlov SM, Oleinik OA. Homogenization of differential operators, Nauka, Moscow 1993 (in Russian).

[23] Allaire G, Bonnetier E, Francfort G, Jouve F. Shape optimization by the homogenization method. Numer Math 1997; 76: 155-92.

[24] Nazarov SA, Sokolowski J. Self adjoint extensions of differential operators in application to shape optimization. CR Mecanique 2003; 331: 667-72.

[25] Nazarov SA, Sokolowski J. Self adjoint extensions for the Neumann Laplacian and applications. Acta Math Sinica 2006; 22: 879906.

[26] Mazja WG, Nazarov SA, Plamenevskii BA. Asymptotic theory of elliptic boundary value problems in singularly perturbed domains, Vol.1, Birkhäuser-Verlag, Basel 2000.

[27] Van Dyke MD. Perturbation methods in fluid mechanics, Academic Press, New York 1964.

[28] Il'in AM. Matching of Asymptotic expansions of solutions of boundary value problems. Trans Math Monogr 102, AMS, Providence, RI 1992.

[29] Ammari H, Kang H. High-order terms in the asymptotic expansions of the steady-state voltage potentials in the presence of conductivity inhomogeneities of small diameter. SIAM J Math Anal 2003; 34: 1152-66.

[30] Pólya G, Szegö G. Isoperimetric inequalities in mathematical physics, Princeton University Press, Princeton, NJ 1951.

[31] Maz'ya VG, Nazarov SA. The asymptotic behavior of energy integrals under small perturbations of the boundary near corner points and conical points. Trans Mosc Math Soc 1988; 50: 77-127.

[32] Argatov II, Sokolowski J. Asymptotics of the energy functional of the Signorini problem under a small singular perturbation of the domain. Comput Math Math Phys 2004; 43: 710-24. 
[33] Argatov II. Introduction to asymptotic modelling in mechanics, polytechnics, St. Petersburg 2004 (in Russian).

[34] Argatov II. The pressure of a punch in the form of an elliptic paraboloid on an elastic layer of finite thickness. J Appl Math Mech 2001; 65: 495-508.

[35] Argatov II, Nazarov SA. Energy release caused by the kinking of a crack in a plane anisotropic solid. J Appl Math Mech 2002; 66: 491-503.
[36] Argatov II, Sabina FJ. Acoustic diffraction by a finite number of small soft bodies. Wave Motion 2008; 45: 238-53.

Received: March 04, 2008

Revised: March 20, 2008

Accepted: March 31, 2008

(C) I.I. Argatov; Licensee Bentham Open.

This is an open access article distributed under the terms of the Creative Commons Attribution License (http://creativecommons.org/licenses/by/2.5/), which permits unrestrictive use, distribution, and reproduction in any medium, provided the original work is properly cited. 